\title{
Valoración y pronóstico de los tumores renales quísticos
}

\author{
J. López Ferrandis, J. Rioja Zuazu, A. Saiz Sansi, J.Mํㅗㄹ Regojo Balboa, J.M. Fernández Montero, \\ D. Rosell Costa, J. E. Robles García, J.J. Zudaire Bergera, J. María Berián Polo
}

Departamento de Urología. Clínica Universitaria de Navarra. Pamplona.

Actas Urol Esp 2005; 29 (1): 74-81

\section{RESUMEN}

VALORACIÓN Y PRONÓSTICO DE LOS TUMORES RENALES GUÍSTICOS

Objetivo: Valorar las características clínicas y patológicas de los tumores renales quísticos tratados en nuestro centro.

Material y métodos: Se realiza una revisión retrospectiva de 239 nefrectomías comparando las variables clínico-patológicas de los tumores quísticos con los tumores renales sólidos. Se analiza la supervivencia en ambos grupos.

Resultados: Los tumores renales quísticos se comportan en nuestra experiencia como los tumores renales sólidos, sin presentar diferencias en la supervivencia. Las variables estudiadas muestran diferencias estadísticamente significativas en el grado histológico y la multiplicidad, siendo los tumores quísticos de menor grado y más frecuentemente múltiples.

Conclusiones: El comportamiento de los tumores renales quísticos no es diferente al de los sólidos.

Palabras clave: Tumor renal. Quístico. Pronóstico.

\section{ABSTRACT}

VALUATION AND PROGNOSTIC OF CYSTIC RENAL TUMORS

Objetive: To evaluate the clinical and pathological characteristics of cystic renal tumors in our center.

Material and methods: A retrospective review of 239 nephrectomies is performed comparing the clinical and pathological variables of cystic tumors with those of solid renal tumors. Survival outcomes are analyzed in both groups.

Results: Our experience shows that cystic renal tumors behave like solid renal tumors, with no differences in survival shown. The variables studied show statistically significant differences in histological grade and number of tumors, with cystic tumors having a lower histological grade and being more often multiple in number.

Conclusions: The behavior of cystic renal tumors is no different than that of solid renal tumors.

Key words: Renal tumor. Cystic. Prognosis.
\end{abstract}

$\mathrm{M}$ ás del $20 \%$ de los adultos poseen quistes renales, en su mayoría quistes serosos simples, en los estudios de imagen no invasivos ${ }^{1-3}$. Este porcentaje aumenta en las autopsias hasta en un 50\% en las personas mayores de 50 años $^{2,4}$.

Las masas renales quísticas continúan siendo hoy en día un reto diagnóstico, debido a la dificultad para establecer criterios claros que diferencien las masas quísticas tumorales de las benignas. Se clasifican como unilocular, multilocular o quístico con necrosis.

El cáncer renal de tipo quístico corresponde entre un 2,3-15\% $\%^{3,5-8}$ de los tumores malignos de riñón, en los que se distingue en el examen histopatológico cavidades quísticas únicas o múltiples, llenas de líquido, contorneadas por una o más capas de células que se asemejan a las células claras del túbulo epitelial renal ${ }^{9}$.
El carcinoma quístico renal (CQR) es habitualmente diploide ${ }^{10}$, de bajo grado ${ }^{4,10}$ y estadio. Su proliferación es lenta $1^{0,11}$ y para algunos autores ${ }^{9}$ no presenta metástasis. Es por tanto un tumor renal de mejor pronóstico y supervivencia más larga ${ }^{11}$.

\section{MATERIAL Y MÉTODOS}

Se ha realizado una revisión retrospectiva de las historias clínicas de las 239 nefrectomías realizadas en nuestro Centro entre enero de 1995 y diciembre de 2002. Se estudian los 21 pacientes a los que se intervino por presentar quiste renal complejo en las pruebas de imagen. De las 21 nefrectomías realizadas, 2 pacientes no presentaron tumor en la pieza de anatomía patológica. Uno de los pacientes presentaba un quiste renal de tipo IIb en la clasificación de Bosniak, con 
aumento del tamaño y cambios en la morfología durante su control evolutivo, en el que no se encontró tumor en la pieza quirúrgica. El segundo caso se trata de una paciente que presentaba una imagen quística renal con áreas sólidas en su interior, que se realzaba tras la administración de contraste, descubierta de forma casual durante las exploraciones complementarias en el seguimiento de un tumor primario de pulmón. En este caso la anatomía patológica mostró que se trataba de un quiste hidatídico renal.

El grupo de pacientes en los que se demostró CQR se compone de 17 hombres y 2 mujeres con una edad media de 55 años (rango 28-75). Realizaron consulta por sintomatología urológica 9 pacientes, mientras que 10 de los pacientes se diagnosticaron de forma casual en el curso de una revisión o durante las exploraciones complementarias realizadas por otra patología. Los pacientes sintomáticos consultaron por dolor en 4 casos, hematuria en 3 casos, infección en 1 caso y un paciente presentó más de 2 síntomas.

Sólo 11 pacientes presentaron ecografía renal sospechosa de tumor quístico renal, puesto que en 8 casos la tomografía computerizada (TC), realizada por otro motivo, puso en evidencia el quiste sospechoso.

En 18 de los pacientes se realizó TC y en uno de ellos la confirmación diagnóstica y el estadiaje se realizó mediante resonancia magnética por tratarse de un paciente alérgico al contraste iodado.

La TC, y en un paciente la resonancia magnética, ha sido la prueba utilizada para la clasificación y estadificación de los quistes renales.

Para el estudio del estadio clínico, se revisaron los pacientes intervenidos con anterioridad a 1997 y se recodificó el estadio según la Clasificación TNM de la UICC de 1997. Se estadiaron clínicamente como T1 en 13 pacientes, T2 en 4 pacientes y T3 en 2 pacientes.

De los 19 pacientes sólo uno mostraba afectación ganglionar y metástasis a distancia en las pruebas previas a la cirugía, al que se realizó nefrectomía previa al tratamiento inmunoterápico.

Presentaron tumor múltiple 4 pacientes, de los cuales en 2 los tumores se presentaron en el mismo riñón y en otros 2 se diagnosticaron tumores sincrónicos bilaterales.
Se realizó nefrectomía ampliada a 13 pacientes y nefrectomía parcial a 6 pacientes.

El tamaño tumoral presentó una media de $6,19 \pm 3,6 \mathrm{~cm}$ con una mediana de $5,50 \mathrm{~cm}$ (rango 1,50-17).

La anatomía patológica mostró carcinoma de células claras en 14 pacientes, carcinoma cromófobo 1 paciente, carcinoma papilar en 3 pacientes y nefroma quístico en 1 paciente. Mostraban un grado histológico G1 10 pacientes, G2 7 pacientes y G3 1 paciente.

El estadio patológico fue pT1 en 11 pacientes, pT2 en 5 pacientes y pT3 en 3 pacientes. De los 13 pacientes a los que se realizó linfadenectomía, sólo 2 presentaron afectación patológica ganglionar.

Media de seguimiento 20,1 meses con una mediana de 15,8 meses.

Dos pacientes han presentado progresión de la enfermedad. Han fallecido durante el seguimiento 2 pacientes, aunque sólo uno de ellos por causa tumoral.

Se muestran las variables más relevantes de los tumores quísticos en la Tabla 1.

El análisis estadístico se ha realizado mediante el programa SPSS.

Para el estudio de asociación de variables cualitativas, se han utilizado tablas de contingencia 2xb, determinándose la chi-cuadrado de Pearson o el test exacto de Fisher.

Para el estudio de variables cuantitativas, las medias se han comparado mediante la prueba $t$ para la comparación de medias o la U de MannWhitney según sigan o no una distribución normal.

Para el estudio de la supervivencia actuarial se han realizado curvas de Kaplan-Meier.

\section{RESULTADOS}

No se encuentran diferencias respecto a la edad, el sexo, la clínica, el diagnóstico casual, el estadio clínico, la localización (lateralidad) ni la presencia de metástasis en el momento del diagnóstico entre el grupo de pacientes con CQR y el grupo de pacientes con carcinoma renal sólido.

Se encuentran diferencias estadísticamente significativas respecto a la multiplicidad $(\mathrm{p}=0,007)$, encontrando mayor porcentaje de tumores múltiples en el grupo de pacientes con CQR. 
Tabla 1

Aspectos más relevantes de los tumores quísticos

\begin{tabular}{|c|c|c|c|c|c|c|}
\hline & Clínica & Bosniak & $\begin{array}{l}\text { Tratamiento } \\
\text { (nefrectomia) }\end{array}$ & $\begin{array}{l}\text { Tipo } \\
\text { celular }\end{array}$ & $\begin{array}{c}\text { Grado } \\
\text { pat }\end{array}$ & pT \\
\hline 1 & hematuria & 4 & ampliada & Cs claras & I & pT1 \\
\hline 2 & asintomático & 4 & ampliada & papilar & I & pT1 \\
\hline 3 & asintomático & 3 & ampliada & papilar & II & pT2 \\
\hline 4 & dolor & 3 & ampliada & Cs claras & I & pT1 \\
\hline 5 & asintomático & 3 & ampliada & Cs claras & I & pT1 \\
\hline 6 & asintomático & 3 & ampliada & Cs claras & II & pT3 \\
\hline 7 & dolor & 3 & parcial & Cs claras & I & pT1 \\
\hline 8 & dolor & 3 & ampliada & Cs claras & II & pT2 \\
\hline 9 & infección & $2 \mathrm{~b}$ & ampliada & papilar & II & pT1 \\
\hline 10 & dolor & 3 & parcial & Cs claras & I & pT2 \\
\hline 11 & asintomático & 3 & parcial & cromófobo & II & pT1 \\
\hline 12 & asintomático & 3 & parcial & Cs claras & I & pT1 \\
\hline 13 & hematuria & 4 & ampliada & Cs claras & I & pT3 \\
\hline 14 & hematuria & 4 & parcial & nefroma & - & pT1 \\
\hline 15 & asintomático & 4 & ampliada & Cs claras & II & pT2 \\
\hline 16 & asintomático & 4 & parcial & Cs claras & I & pT1 \\
\hline 17 & asintomático & 3 & ampliada & Cs claras & II & pT2 \\
\hline 18 & asintomático & 4 & ampliada & Cs claras & I & pT1 \\
\hline 19 & Más de dos & 4 & ampliada & Cs claras & III & pT3 \\
\hline
\end{tabular}

No hay diferencias respecto al tipo de cirugia realizado.

No existen diferencias respecto al tamaño ni el estadio patológico $(\mathrm{p}=0,3)$.

Hay diferencias respecto al diagnóstico anátomo-patológico $(\mathrm{p}=0,005)$, pero cuando se recodifica el tipo histopatológico en cs claras vs resto, no se encuentran diferencias estadísticamente significativas $(\mathrm{p}=0,79)$.

Sí existen diferencias respecto al Grado patológico 1-2 vs 3-4 ( $\mathrm{p}=0,026)$.

La comparación de porcentajes entre los tumores quísticos y sólidos se muestra en la Tabla 2.

Las curvas de supervivencia no muestran diferencias significativas en cuanto a la supervivencia libre de enfermedad entre ambos grupos (Log Rango =0,45) (Fig. 1).

\section{COMENTARIOS}

La naturaleza del CQR todavía no está claramente delineada ${ }^{10}$.

Adsay et al. ${ }^{12}$ formulan la teoria de un mecanismo patogénico común para los tumores quísticos renales y los tumores
Tabla 2

Variables clínicas y patológicas de los tumores renales quísticos y sólidos

\begin{tabular}{|c|c|c|c|c|}
\hline & & T. Quísticos & T. Sólidos & $\mathbf{p}$ \\
\hline EDAD & & $55 \pm 15$ & $60,8 \pm 44$ & N S \\
\hline SEXO & $\begin{array}{l}\text { hombre } \\
\text { mujer }\end{array}$ & $\begin{array}{c}17(89,5 \%) \\
2(10,5 \%)\end{array}$ & $\begin{array}{c}167(77 \%) \\
51(23 \%)\end{array}$ & N S \\
\hline Dx CASUALSí & $\begin{array}{c}10(52,6 \%) \\
\text { no }\end{array}$ & $\begin{array}{c}110(50,4 \%) \\
9(47,4 \%)\end{array}$ & $\begin{array}{c}\text { N S } \\
108(49,6 \%)\end{array}$ & \\
\hline MULTIPLICIDAD & $\begin{array}{l}\mathrm{T} \text { único } \\
\mathrm{T} \text { múltiple }\end{array}$ & $\begin{array}{c}15(79 \%) \\
4(21 \%)\end{array}$ & $\begin{array}{c}210(96,4 \%) \\
8(3,6 \%)\end{array}$ & 0,007 \\
\hline $\begin{array}{l}\text { ESTADIO } \\
\text { CLÍNICO }\end{array}$ & $\begin{array}{l}\text { T } 1-2 \\
\text { T } 3-4\end{array}$ & $\begin{array}{c}17(89,5 \%) \\
2(10,5 \%)\end{array}$ & $\begin{array}{c}189(87 \%) \\
29(13 \%)\end{array}$ & N S \\
\hline TAMAÑO & & $6,2 \pm 3,6$ & $6,3 \pm 3,2$ & N S \\
\hline $\begin{array}{l}\text { ESTADIO } \\
\text { PATOLÓGICO }\end{array}$ & $\begin{array}{l}\text { pT } 1-2 \\
\text { pT } 3-4\end{array}$ & $\begin{array}{l}16(84 \%) \\
3(16 \%)\end{array}$ & $\begin{array}{c}139(64 \%) \\
79(36 \%)\end{array}$ & N S \\
\hline $\begin{array}{l}\text { TIPO } \\
\text { HISTOLÓGICO }\end{array}$ & $\begin{array}{l}\text { Cs claras } \\
\text { Resto }\end{array}$ & $\begin{array}{c}14(74 \%) \\
5(26 \%)\end{array}$ & $\begin{array}{c}154(71 \%) \\
64(29 \%)\end{array}$ & N S \\
\hline $\begin{array}{l}\text { GRADO } \\
\text { TUMORAL }\end{array}$ & $\begin{array}{c}\text { G } 1-2 \\
\text { G } 3-4 \\
\text { perdido }\end{array}$ & $\begin{array}{c}17(89,4 \%) \\
1(5,3 \%) \\
1(5,3 \%)\end{array}$ & $\begin{array}{c}138(63,3 \%) \\
6530 \%) \\
15(6,7 \%)\end{array}$ & 0,026 \\
\hline METÁSTASIS & $\begin{array}{l}\text { No } \\
\text { Sí }\end{array}$ & $\begin{array}{c}18(94,7 \%) \\
1(5,3 \%)\end{array}$ & $\begin{array}{c}182(83,5 \%) \\
36(16,5 \%)\end{array}$ & N S \\
\hline
\end{tabular}




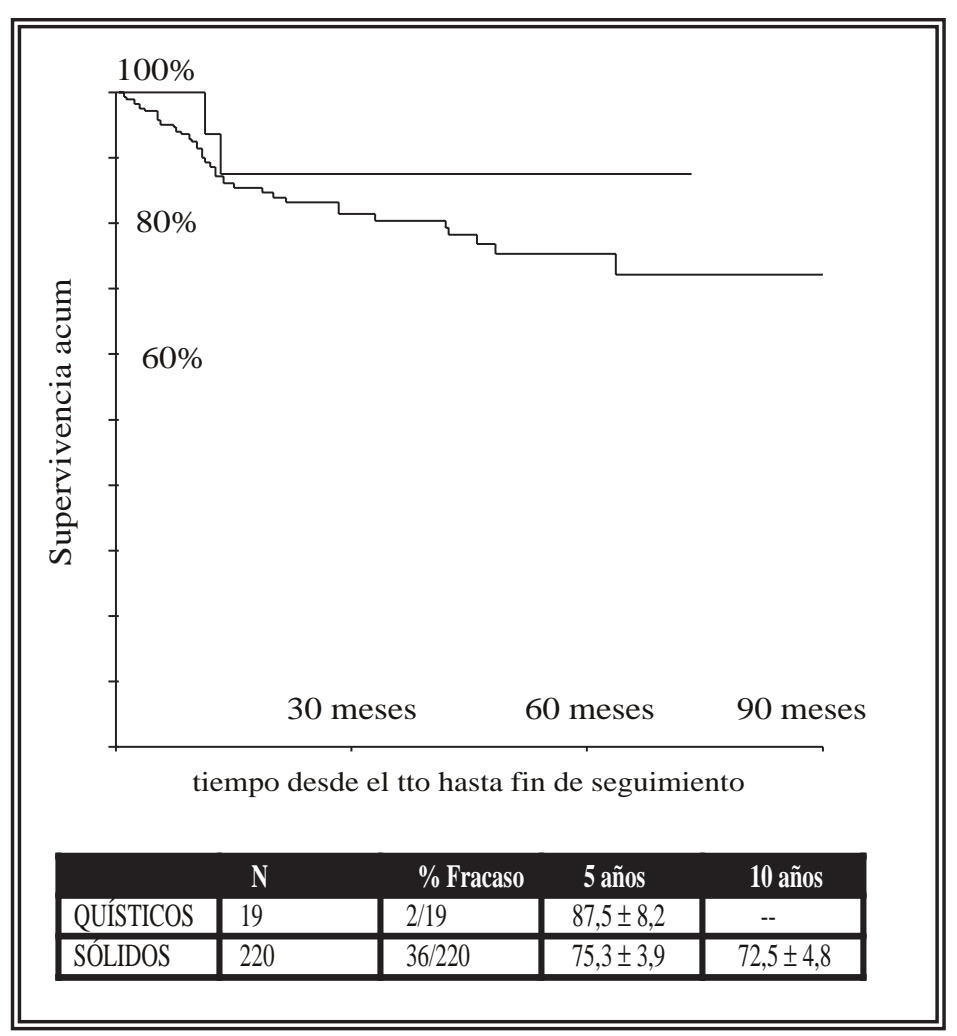

FIGURA I. Curvas de supervivencia. Tumores renales quísticos VS tumores renales sólidos.

mucinosos del páncreas y el hígado, que puede estar relacionado con el mesénquima periductal fetal debido al paralelismo clínico y patológico entre éstos. Gibson relaciona quiste con tumor respecto a su situación topográfica, siendo Hartman ${ }^{13}$ quien propone los mecanismos patológicos implicados en la génesis de tumores renales quísticos:

1. Tumor renal originado en el epitelio de revestimiento de un quiste renal.

2. Cambios quísticos dentro del tumor, generalmente debidos a importante necrosis del mismo. La necrosis tumoral daría lugar a la formación de una cavidad quística uniloculada rellena de restos necróticos y hemáticos.

3. Crecimiento quístico intrínseco unilocular (cistoadenocarcinoma). Habitualmente de tipo papilar y las células revisten completamente las paredes del quiste.

4. Crecimiento quístico intrínseco multilocular. Múltiples quistes no comunicados entre si, rellenos de material hemático. Las células tumorales, habitualmente del tipo de células claras revisten de modo continuo las paredes de los lóculos. Un 40\% de los tumores renales quísticos corresponden a este grupo.

Todavía permanece incierto si el carcinoma renal surge de un quiste preexistente o si el quiste es parte del crecimiento tumoral, en cualquier caso, los pacientes con enfermedad quística adquirida renal tienen un alto riesgo de padecer un carcinoma renal ${ }^{9}$.

Los quistes con frecuencia sufren cambios como hemorragia o infección; el CQR debe presentar un patrón de crecimiento quístico de novo (uni o multilocular) en el que la necrosis no es infrecuente ${ }^{7}$.

Stephen et al. ${ }^{14}$ describen el carcinoma renal quístico multilocular como lesiones multiquísticas bien demarcadas que contienen agregados de tamaños variables de células claras neoplásicas, que muestran grado nuclear I y pocas o ninguna actividad mitótica. En estos, no se ha observado nunca invasión vascular o transformación sarcomatoide ni se ha publicado ningún caso de metástasis a distancia.

Para Madrid et al. ${ }^{15}$ el nefroma quístico multilocular es un tumor de comportamiento benigno que habitualmente no produce sintomatología. Se trata de una lesión compuesta enteramente por quistes y sus septos, el tumor conforma una masa bien diferenciada del resto del parénquima renal, los quistes están tapizados por epitelio plano o cuboidal, los septos son la única porción sólida de la neoplasia conformando la pared externa de los quistes sin expansiones nodulares sólidas y están compuestas por tejido fibroso, en el cual puede existir un pequeño número de túbulos bien diferenciados o de células del blastema en los septos, aunque si han observado la existencia de tejido maduro heterólogo como músculo esquelético.

Ante una masa quística renal, se debe de plantear el diagnóstico diferencial con diversas entidades que se relacionan en la Tabla 3.

Las lesiones quísticas pueden presentar hallazgos que sugieran patología maligna, siendo la clasificación descrita por Bosniak en 1986 la más aceptada como ayuda en el manejo de masas quísticas renales complicadas ${ }^{16}$ (Tabla 4). 
Tabla 3

Diagnóstico diferencial de masa renal multiquística. Entidades a tener en cuenta en el diagnóstico diferencial según Hartman ${ }^{2,6}$.

1. Neoplasia

A. Origen tubular

Carcinoma renal

Adenacarcinoma renal

B. Originados en el blastema

Nefroma quístico multilocular (cistoadenoma renal)

2. Enfermedad quística

A. Enfermedad segmentaria quística

B. Septos intraquísticos

C. Enfermedad multiquística segmentaria

3. Inflamaciones
A. Quiste infectado
B. Xantogranulomatosis segmentaria
C. Malakoplaquia
D. Hidatidosis

4. Traumatismos

Hematoma organizado

5. Vascular

Malformaciónes arteriovenosas y linfáticas

Tabla 4

Clasificación de Bosniak para masas renales quísticas

\begin{tabular}{|c|c|c|}
\hline Lesión & Caracteristicas & Interpretación \\
\hline Tipo 1 & Quiste simple benigno & No más estudios ni $\mathrm{Tt}^{\circ}$ \\
\hline Tipo 2 & $\begin{array}{l}\text { Quiste mínimamente complicado } \\
\text { Calcificación mural mínima } \\
\text { septos finos, contenido hiperdenso }\end{array}$ & Riesgo mínimo \\
\hline Tipo $2 \mathrm{~F}$ & $\begin{array}{l}\text { Varias características del tipo } 2 \text { ó } \\
\text { Algunas del tipo } 3\end{array}$ & Seguimiento estrecho \\
\hline Tipo 3 & $\begin{array}{l}\text { Presencia de signos sugestivos } \\
\text { aunque no definitivos de malignidad } \\
\text { tabiques gruesos o nodulares } \\
\text { calcificación grosera e irregular } \\
\text { nodularidad o engrosamiento de la } \\
\text { pared densidad heterogénea }\end{array}$ & $\begin{array}{l}\text { Malignidad en } 50 \% \\
\text { control estrecho ó } \\
\text { Ttº quirúrgico }^{\circ}\end{array}$ \\
\hline Tipo 4 & $\begin{array}{l}\text { Neoplasia quística } \\
\text { Áreas claras de realce }\end{array}$ & $\mathrm{Tt} \mathrm{t}^{\circ}$ quirúrgico \\
\hline
\end{tabular}

Tabla 5

Características ecográficas de quiste seroso simple ${ }^{1}$.

1. Tratarse de una masa renal sin ecos en su interior.

2. Presentar una buena transmisión de los ecos y tener refuerzo posterior.

3. Poseer bordes netos, finos y lisos.
En la clasificación de Bosniak, la proporción de pacientes con carcinoma renal aumenta a medida que aumenta el grado de clasificación ${ }^{7}$.

El estudio de las masas renales se fundamenta en la actualidad en la ecografía y la $\mathrm{TC}^{3}$. La ecografía permite diferenciar fácilmente una masa sólida de una quística.

La ecografía, como la TC, es un método diagnóstico adecuado para caracterizar las masas quísticas que no se ajustan a los criterios de quiste renal simple ${ }^{5}$ (Tabla 5). De modo concebible, la interfase entre líquido y sólido creada por el componente fluido del quiste aumentan la sensibilidad de las técnicas de imagen, aumentando el diagnóstico incidental del tumor con menor estadio que en el caso de tumores sólidos ${ }^{9}$.

La ecografía demuestra uno o más septos por dentro del quiste. Estos septos pueden ser el resultado de dos quistes compartiendo una pared común o la manifestación de la organización de un quiste que se ha complicado con hemorragia o infección. Todos los septos deben ser delgados $(<1 \mathrm{~mm})$, lisos y pegados a la pared sin asociar elementos espesos. Cuando existen calcificaciones finas en los septos o espesamiento de la pared o una masa quística renal no se ajusta a los criterios de quiste renal simple, se hacen obligatorias las exploraciones complementarias $^{5}$, habitualmente mediante $\mathrm{TC}^{8}$.

La TC pone de manifiesto la heterogenicidad y las características densitométricas de la masa y evalúa con mayor precisión que la ecografía el contorno de la pared quística ${ }^{8}$.

La sensibilidad diagnóstica de la TC para diferenciar una masa sólida de una quística o de un tumor de crecimiento intraparietal quístico está próxima al 100\%. El engrosamiento e irregularidad de la pared, la heterogenicidad del contenido, la calcificación de la pared y la apariencia multilocular o septada son los hallazgos que con mayor frecuencia acompañan a las formas quísticas de las neoplasias renales $^{23}$.

Características sugestivas de neoplasia para Dalla Palma y cols ${ }^{17}$ son la 
presencia de septos intraquísticos gruesos, alta ganancia tras la administración de contraste y la presencia de vegetaciones intraquísticas, siendo las dos últimas las de mayor valor diagnóstico.

La resonancia nuclear no aporta mayor sensibilidad ni rentabilidad diagnóstica que la TC.

Tanto en la radiografia simple de abdomen como en la urografia endovenosa se puede apreciar efecto masa del riñón afecto. Entre un 20$25 \%$ de carcinomas renales quísticos pueden presentar calcificaciones, siendo éstas curvilineas y periféricas, aunque pueden aparecer calcificaciones distróficas ${ }^{8}$.

La arteriografía, aunque es un método diagnóstico poco utilizado en la actualidad, puede utilizarse en casos dudosos mediante ecografía o TC 5 .

Durante la década de los 70 la diferenciación entre quiste y carcinoma renal no era siempre aparente, por lo que la punción del quiste y el aspirado del fluido del mismo (PAAF), se realizaba como un test diagnóstico estándar. Hoy día, el papel de la PAAF es limitado ${ }^{4,5,7}$ y dudoso ${ }^{8}$ y gracias al avance en las técnicas de imagen no invasivas, estos procedimientos son cada vez menos necesarios. En los quistes tumorales, el estudio del líquido aspirado por PAAF mostrará aumento de la $\mathrm{LDH}$, colesterol y proteínas, así como un aspecto turbio o hemático ${ }^{3}$. La citología del líquido aspirado, sólo es positiva en un 14\% de los $\operatorname{casos}^{18}$, y además la PAAF no debe ser utilizada en casos de quistes multiloculares, puesto que cada quiste debe ser evaluado y un resultado negativo no descarta malignidad ${ }^{4,5,7}$.

Nuevos análisis como la cuantificación de IL6, así como el factor de crecimiento de fibroblastos pueden ayudar al diagnóstico preoperatorio de CQR y predecir las características biológicas $^{18}$, pero probablemente se necesiten nuevos estudios para que el aspirado por punción de los quistes presente un claro beneficio diagnóstico para una técnica invasiva no exenta de riesgos.

Los estudios mediante ecografia, TC, arteriografía, PAAF y resonancia no han resuelto el problema de diferenciar masas multiloculadas benignas y malignas ${ }^{2}$. Aunque la sensibilidad de las técnicas de imagen ha aumentado en los últimos años, el diagnóstico de carcinoma quístico renal no siempre es posible ${ }^{3,7}$. El diagnóstico certero preoperatorio de las masas quísticas es con frecuencia difícil ${ }^{4,11,18,19}$ y ningún método diagnóstico es absolutamente fiable a la hora de catalogar una masa renal quística compleja ${ }^{6,11,20}$. Es generalmente dificil diferenciar el CQR de las lesiones quísticas benignas. En muchos casos el diagnóstico definitivo sólo puede realizarse mediante el estudio histopatológico ${ }^{10,11,15}$. Incluso si se realiza una biopsia peroperatoria se puede obtener un falso negativo ${ }^{2,3}$, puesto que puede no encontrarse tumor en la zona de la pared biopsiada ${ }^{8}$. Ni la ausencia de síntomas ni el aspecto quístico de una masa son datos tranquilizadores respecto a su naturaleza ${ }^{6}$. Ante una masa quística que no reúna los criterios de quiste simple, podemos estar ante un proceso neoplásico y debemos realizar exploraciones complementarias y cirugía ante la menor duda ${ }^{2,6}$.

Bosniak recomienda no tratar los quistes de categorías I y II y si categorías III y IV. Muchos autores son de la opinión que la categoría III también debe ser tratada quirúrgicamente, pero los estudios de imagen, en ocasiones, no estiman con suficiente sensibilidad el espesamiento e irregularidad, la calcificación o reptación, por lo que la distinción entre las categorías II y III puede reportar dificultades 7 .

Sólo en 11 pacientes se realizó ecografía puesto que en 8 de ellos el hallazgo de un quiste renal complejo se observó durante la exploración mediante TC realizada por otro motivo. Los 11 pacientes identificados por ecografía fueron valorados posteriormente con TC para confirmar el diagnóstico, con la excepción de un paciente alérgico al contraste iodado al que se le realizó una resonancia.

La TC mostró lesiones sugestivas de quiste renal complejo en 21 pacientes de los que en 19 se confirmó la existencia de tumor. Un paciente no presentó tumor en el estudio anatomopatológico y en otro paciente se mostró la existencia de un quiste hidatídico renal. El paciente que no mostró lesiones tumorales en la pieza quirúrgica presentaba cambios morfológicos en el control evolutivo. Así, la TC se muestra por si misma un método diagnóstico adecuado para identificar las lesiones quísticas y decidir una actitud quirúrgica.

La exploración quirúrgica mediante nefrectomía parcial es, por el momento, el proceder de 
mayor fiabilidad diagnóstica y terapéutica ante masas renales quísticas de etiología incierta ${ }^{8}$. Para algunos autores ${ }^{7,11,21}$ se debe indicar nefrectomía parcial debido al bajo potencial de estos tumores ${ }^{4}$. La nefrectomía parcial se ha demostrado efectiva en los carcinomas renales quísticos multiloculares menores de $4 \mathrm{~cm}$ de diámetro sin penetración capsular en la biopsia peroperatoria $^{11,14}$. Aunque los pacientes tratados mediante nefrectomía parcial presentan una recurrencia en torno al 6-15\% y algunos mueren por metástasis en los primeros 5 años ${ }^{9}$, en general, y como en el tratamiento de los tumores renales sólidos, se acepta la nefrectomía parcial para aquellos casos con riñón único o aquellos con función renal disminuida ${ }^{10}$, así como para tumores menores de $4 \mathrm{~cm}$.

La biopsia laparoscópica del carcinoma renal quístico seguida de cirugía abierta parece no aumentar la incidencia de siembra peritoneal, recurrencia local ni metástasis a distancia ${ }^{22}$, lo que hace que algunos autores consideren esta alternativa en su proceder diagnóstico y terapéutico.

Los tumores de mayor tamaño y las lesiones que afectan al pedículo vascular hacen imperativa una cirugía más agresiva, justificada ante la duda de presencia de tumor en la lesión. Asimismo, cuando la biopsia peroperatoria confirma la existencia de células tumorales y estas llegan al margen de resección, la nefrectomía radical es el proceder terapéutico de elección.

Las variables clínicas de los tumores quísticos son semejantes a los carcinomas renales sóli$\operatorname{dos}^{3,10,11}$, aunque la presencia de metástasis en el momento del diagnóstico es menor ${ }^{10}$ y suelen ser tumores de bajo grado y estadio ${ }^{10,11,19}$. Para Kirsh y cols ${ }^{23}$ que recientemente han reexaminado lesiones quísticas de células claras del riñón han propuesto que la enfermedad renal multiquística de células claras, representa una forma distinta de neoplasia benigna. Otros autores plantean que el carcinoma quístico multilocular renal es un importante subtipo del carcinoma renal que es distinto del carcinoma sólido renal ${ }^{10}$, asociado a un excelente pronóstico ${ }^{9}$.

En nuestra serie encontramos que los tumores renales sólidos y quísticos comparten las mismas características clínicas y presentan un tamaño similar, aunque a diferencia de lo publicado, en nuestro caso, la presencia de metástasis al diagnóstico no difiere entre ambos grupos. Respecto a las variables patológicas, coincidimos con lo publicado en que son tumores de menor grado, pero en nuestra serie no encontramos diferencias significativas respecto al estadio patológico y por otro lado, los $\mathrm{CRQ}$ tratados en nuestro centro, presentan mayor multiplicidad que los tumores sólidos.

Las series publicadas muestran los CRQ como tumores de baja progresión (1/32 para Cloix y cols $^{5}$ y 0/24 para Corica y cols ${ }^{9}$ ), así como baja incidencia de metástasis en el momento del diagnóstico (1/21 para Koga y $\operatorname{cols}^{10}$ y 0/32 para Cloix y cols ${ }^{5}$ ) por lo que se asocian a mejor pronóstico ${ }^{19}$.

La revisión que hemos realizado no muestra que los tumores quísticos presenten mejor pronóstico que los sólidos. Los CRQ tratados en nuestro centro muestran un estadio patológico similar al de los sólidos, quizás influenciado por el incremento del diagnóstico incidental, pero con mayor frecuencia de multiplicidad.

\section{CONCLUSIONES}

En nuestra experiencia, los CQR presentan mayor multiplicidad y menor grado histológico, siendo el resto de las variables clínicas y patológicas estudiadas así como la supervivencia similares a los tumores sólidos.

\section{REFERENCIAS}

1. Zungri E, Tardaguila F, Pereiro M, Pesqueira D, De La Fuente A. Valor de la LDH en el diagnóstico del cáncer renal pseudoquístico. Actas Urol Esp. 1989;13(3):217-218.

2. Puyol M, Méndez V, Gómez J. Adenocarcinoma quístico renal. Actas Urol Esp. 1993;17(9):603-607.

3. Gutierrez JL, Martín B, Hernández R et al. Adenocarcinoma quístico de riñón. A proposito de 18 casos. Arch Esp Urol. 1996;49(6):573-579.

4. Hayakawa M, Hatano T, Tsuji A, Nakajima F, Ogawa Y. Patients with renal cysts associated with renal cell carcinoma and the clinical implications of cyst puncture: A study of 223 cases. Urology 1996;47(5):643-646.

5. Cloix P, Martin X, Pangaud C, et al. Surgical management of complex renal cysts: A series of 32 cases. J Urol 1996;156: 28-30.

6. Carcamo P, Moreno JA, Picazo ML, et al. Adenocarcinoma renal quístico multilocular. Arch Esp de Urol. 1993;46(3): 240-244. 
7. Koga S, Nishikido M, Inuzuka S, et al. An evaluation of Bosniak's radiological classification of cystic renal masses. BJU Int. 2000;86:607-609.

8. Calahorra FJ, Pérez C, Passas J, et al. Carcinoma renal quístico: presentación de cinco casos. Actas Urol Esp. 1993;17(9):614-622.

9. Corica FA, Iczkowski KA, Cheng L, et al. Cystic renal cell carcinoma is cured by resection: A study of 24 cases with long-term followup. Urology. February 1999;161:408-411.

10. Koga S, Nishikido M, Hayashi T, Matsuya F, Saito Y, Kanetake H. Outcome of surgery in cystic renal cell carcinoma. Urology. 2000;56(1):67-70.

11. Bielsa O, Lloreta J, Gelabert-Mas A. Cystic renal cell carcinoma: pathological features, survival and implications for treatment. Br J Urol. 1998;82(1):16-20.

12. Adsay NV, Eble JN, Srigley JR, Jones EC, Grignon DJ. Mixed epithelial and stromal tumor of the kidney. Am J Surg Pathol. 2000;24(7):958-70.

13. Hartman DS, Davis CH J Jr, Johns T, Goldman SM. Cystic renal cell carcinoma. Urology 1986;28:145.

14. Stephen G, Weiss II, Reza G Hafez, David T Uehling. Multilocular cystic renal cell carcinoma: Implications for nephron sparing surgery. Urology 1998;51(4):635-637.

15. Madrid FJ, Serrano I, Rivas JA, et al. Nefroma quístico multilocular. Aportación de un caso con referencia a los cambios ecográficos observados. Arch Esp Urol. 1998;51 (5):493-8.

16. Sánchez D, López J, Arocena J et al. Clínica, diagnóstico y pronóstico del carcinoma renal. Actas Urol Esp. 2002;26 (8):532-540.

17. Dalla-Palma L, Pozzi F, Donna A et al. Cystic renal tumors: US and CT findings. Urol Radiol. 1990;12:67.
18. Hayakawa M, Nakajima F, Tsuji A, Asano T, Hatano T, Nakamura H. Citokine levels in cystic renal masses associated with renal cell carcinoma. Urology 1998;159:14591464.

19. Onishi T, Oishi Y, Goto H, Tomita M, Abe K, Sugaya S. Cyst associated renal cell carcinoma: clinicopathologic characteristics and evaluation of prognosis in 27 cases. 2001 Jun;8(6):268-274.

20. Garrido C, Sanroma I, Garmendia JC, Ruiz I, López JA, Arocena F. Adenocarcinoma renal quístico. Arch Esp Urol. 1990;43(9): 1015-1018.

21. Matsushita Y, Suzuki K, Tamura T, Maeda K, Fujioka T. Cystic renal cell carcinoma: report of four cases. Hinyokika Kiyo. 1997;43(10):719-22.

22. Santiago L, Yamaguchi R, Kaswick J, Bellman GC. Laparoscopic management of indeterminate renal cysts. Urology. 1998;52(3):379-383.

23. Kirsh EJ, Straus FH, Goldfischer ER et al. Bening adenomatous multicystic Kidney tumor (Perlmann's tumor) and renal cortical carcinoma with adenomatous multicystic features: 12 cases. Urology 1999;53:65-70.

Dr. J. López Ferrandis

Servicio de Urología

Clínica Universitaria de Navarra

Apartado 4209

31080 Pamplona (Navarra)

(Trabajo recibido el 31 mayo de 2004) 